\title{
Neurophotonics
}

Neurophotonics.SPIEDigitalLibrary.org

\section{The BRAIN Initiative}

\author{
Anna Devor \\ Anna W. Roe \\ Anita Mahadevan-Jansen \\ David A. Boas
}




\title{
The BRAIN Initiative
}

\author{
Anna Devor \\ University of California, San Diego \\ Departments of Neurosciences and Radiology \\ La Jolla, California 92093 \\ and \\ Harvard Medical School \\ Martinos Center for Biomedical Imaging, Massachusetts General Hospital \\ Charlestown, Massachusetts 02129 \\ E-mail: adevor@ucsd.edu
}

\section{Anna W. Roe}

Vanderbilt University

Department of Psychology

Nashville, Tennessee 37240

E-mail: anna.roe@vanderbilt.edu

\section{Anita Mahadevan-Jansen}

Vanderbilt University

Department of Biomedical Engineering

Nashville, Tennessee 37240

E-mail: anita.mahadevan-jansen@vanderbilt.edu

\section{David A. Boas}

Harvard Medical School

Martinos Center for Biomedical Imaging, Massachusetts General Hospital

Charlestown, Massachusetts 02129

E-mail: dboas@nmr.mgh.harvard.edu

What is the first association that comes to mind when you hear "fiber optics"? Connecting one side of the Atlantic Ocean to the other, or high-definition television? For sure, it would not be manipulation of neuronal firing ... unless you are a neuroscientist. While neuroscience has relied on optics to power discovery and advancement from the beginning of time, brain imaging remains an exotic application in mainstream optics and photonics. The Presidential BRAIN Initiative that launched in April of 2013 targets this disconnect by encouraging "hard core" technology experts to step up to the neuroscience quest.

The goal of the Initiative is to develop new technologies driven by the needs of basic and translational neuroscience-easy to say, but difficult to do. The first challenge for the technology expert is to understand the needs of the endpoint application; for the neuroscientist, the challenge is to keep up with the rapidly developing technology and recognize its strengths and limitations.

Generally speaking, brain tissue is an unfriendly environment for optics. Light is scattered by microscopic structures with different refractive indices and absorbed by natural chromophores. Red blood cells fly through capillaries, scattering and absorbing light along the way. The brain moves (slightly but noticeably on a microscopic scale) with respiration and heartbeat beyond what can be corrected for by rigid body registration. Furthermore, the duration of an action potential is only 1-2 ms, which imposes stringent constraints on the acquisition speed required for measurements of brain activity. In addition, brain tissue is sensitive to heating and photodynamic damage, placing limits on photon flux.
Despite these limitations, consider this: arguably the most significant breakthroughs in neuroscience have been powered by advancements in optical technology, from the discovery of synaptic junctions by the father of modern neuroscience Ramon Y. Cajal in the 1880s, to contemporary advances in cell- and system-level neuroscience through utilization of two-photon microscopy, optogenetics, and superresolution microscopy. The BRAIN Initiative seeks to accelerate these efforts by scaling up the development of existing tools and encouraging exploration of new technological concepts to dramatically advance our understanding of how the brain works.

Our first issue of Neurophotonics is published on the upswing of the initiative with a dedicated special section featuring a spectrum of papers on optical tools for measurement and manipulation of the brain's neuronal, metabolic, and hemodynamic activity. This wide scope reflects the breadth of the arena and underscores collaborative opportunities for optics and photonics for targeted technology development rooted in the forefront of neuroscience research.

In short, advances in the brain sciences require the attention of strong technology experts. The BRAIN Initiative has already succeeded in increasing the visibility of this seemingly exotic application within the optical community. The next step is to provide a path for a long-term multidisciplinary relationship enabling comprehensive technological developments emerging from proof of concept studies and leading to broad adoption by basic and clinical neuroscientists. Somewhere along this path, the brain application will become a little less exotic. However, the excitement will only grow as 
application-driven technology and technology-powered neuroscience evolve in a continual dialog.

Anna Devor, $\mathrm{PhD}$, is an associate adjunct professor in the Departments of Neurosciences and Radiology at University of California, San Diego. She also holds an appointment as an instructor in radiology at the Martinos Center for Biomedical Imaging at Massachusetts General Hospital. She is an expert in neuro-vascular-metabolic coupling and regulation of cerebral blood flow. She is known for her innovative application of optical imaging techniques for in vivo imaging of brain activity.

Anna Wang Roe (BA from Harvard 1984, PhD from MIT 1991) is a professor of psychology, biomedical engineering, and radiology at Vanderbilt University. She is also director of the Zhejiang University Interdisciplinary Institute of Neuroscience and Technology (ZIINT) in Hangzhou, China. She is the recipient of Sloan, Whitehall, and Packard Fellowships, is a regular member of $\mathrm{NIH}$ study sections, and holds several editorships for leading neuroscience journals. Her academic interests center on brain function and organization underlying vision and touch, and on development of brain imaging technologies.
Anita Mahadevan-Jansen develops applications of optical techniques for detection of clinical physiology and pathology. Her research at the Vanderbilt Biomedical Photonics Laboratories investigates the applications of optical methods for diagnosis and guidance of therapy, as well as develops infrared neural stimulation (INS) for human clinical and neuroscience applications. She received her bachelor's and master's degrees in physics from the University of Bombay, India, and her master's and $\mathrm{PhD}$ degrees in biomedical engineering from the University of Texas at Austin, USA. She joined the Vanderbilt engineering faculty in 1996. She is currently the Orrin H. Ingram Professor of Biomedical Engineering at Vanderbilt University and holds a secondary appointment in the Department of Neurological Surgery.

David Boas received his $\mathrm{PhD}$ in physics at the University of Pennsylvania in 1996. He is currently a professor of radiology at Massachusetts General Hospital, Harvard Medical School, where he directs the Optics Division at the Martinos Center of Biomedical Imaging. $\mathrm{He}$ is the founding Editor-in-Chief of Neurophotonics. His research focuses on developing novel optical imaging methods to study cerebral physiology and pathophysiology, particularly related to oxygen delivery and consumption. 